DOI 10.35381/cm.v5i9.142

\title{
La percepción de la experiencia de compras en el comercio electrónico, desde un enfoque teórico práctico
}

\section{The perception of the experience of purchases in electronic commerce, from a practical theoretical approach}

\author{
Alfredo Mauricio Astudillo Mamarandi \\ aastudillo@unach.edu.ec \\ Universidad Nacional de Chimborazo Riobamba \\ Ecuador \\ https://orcid.org/0000-0001-5204-4927 \\ Santiago Barriga \\ sbarriga@unach.edu.ec \\ Universidad Nacional de Chimborazo Riobamba \\ Ecuador \\ https://orcid.org/0000-0001-5527-148X
}

Recibido: 19 de abril del 2019

Aprobado: 01 de junio del 2019

\section{RESUMEN}

Uno de los factores primordiales de la investigación es analizar y dilucidar desde un enfoque teórico los factores más importantes que intervienen en el comercio electrónico, entender cómo funciona desde el proceder conceptual, para luego plantear una técnica de recolección de datos Ilamada "Focus Gruop" el cual ayudo a entender de la percepción del usuario en la interacción web, basado en la experiencia de compra virtual y su relación a lo expuesto en el ámbito teórico. Es un estudio no experimental ya que se le trato al fenómeno tal como se presenta en el entorno, para comprender el comportamiento de un usuario WEB frente a tiendas virtuales que ofrecen productos de primera necesidad, demostrando así que los panelistas, en su mayoría mujeres tenían una relación de familiaridad con este tipo de negocios virtuales y que no les era ajeno la navegabilidad en las opciones de estudio que se presentó en la investigación.

Descriptores: Comercio electrónico; sistema de pago; experiencia de compra. 


\author{
CIENCIAMATRIA \\ Revista Interdisciplinaria de Humanidades, Educación, Ciencia y Tecnología \\ Año Vl. Vol. VI. №10. Enero - Julio 2020 \\ Hecho el depósito de ley: pp201602FA4721 \\ ISSN-L: 2542-3029; ISSN: 2610-802X \\ Universidad Nacional Experimental Francisco de Miranda (UNEFM). Santa Ana de Coro. Venezuela \\ Alfredo Mauricio Astudillo Mamarandi; Santiago Barriga; Mayra Gissela Lucio Solano
}

\begin{abstract}
One of the main factors of the research was to analyze and elucidate from a theoretical approach the most important factors involved in electronic commerce, understand how it works from the conceptual procedure, and then propose a data collection technique called "Focus Gruop". which helped to understand the perception of the user in the web interaction, based on the virtual shopping experience and its relation to the exposed in the theoretical field. It is a non-experimental study since the phenomenon is treated as presented in the environment, to understand the behavior of a WEB user in front of virtual stores that offer essential products, thus demonstrating that panelists, mostly women, had a relationship of familiarity with this type of virtual business and that the navigability in the study options presented in the research was no stranger to them.
\end{abstract}

Descriptors: Electronic commerce; payment system; shopping experience.

\title{
INTRODUCCION
}

El mercadeo dentro de la WEB tiene un crecimiento muy vertiginoso, se lo denomina el mercado de las oportunidades, porque no está sujeto a cultura, origen o estatus. Y si, se lo sabe gestionar, es una plataforma eficiente para realizar grandes negocios rentables y exitosos (Gosende, 2014). De igual manera el comercio electrónico, no es más que el uso de medios electrónicos enlazados por la red para efectuar transacciones comerciales de productos y servicios; Toda transacción nace de algo muy primordial que es la necesidad o la insatisfacción de un bien o servicio, el cual lleva a segmentar cada vez más los mercados y también a cortar brechas entre los consumidores. La oferta y la demanda no requieren de un lugar físico necesariamente.

Al igual que un comercio tradicional tienen sus ventajas como desventajas, el comercio electrónico está sujeto al mismo paradigma, sus factores pueden ser muy cambiantes, ya sea por competitividad, manejo de la tecnología, lectura de datos estadísticos o simplemente por canales de comunicación complejas, sin embargo, en los últimos años el mundo ha visto una transformación en la compra y venta de productos y servicios en 


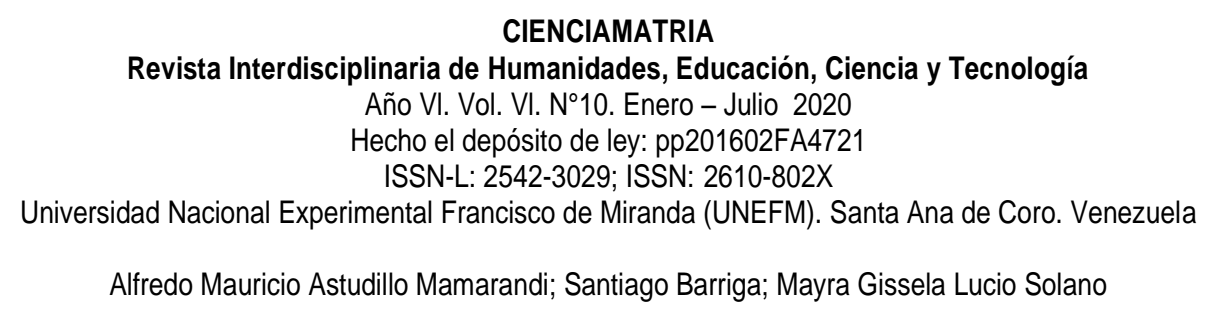

línea. La innovación está marcada por el uso de la tecnología, su rapidez, seguridad y diversidad al momento de escoger una compra, la idea de negocio no siempre es innovadora, pero si es diferenciadora, por las características que posee cada uno de los negocios en línea, por citar un ejemplo, a simple vista se diría que Amazon y $B \& H$, vende lo mismo en cuanto a tecnología y telecomunicaciones, hasta cierto punto se puede coincidir, pero si se analiza desde la percepción del usuario podremos definir diferentes factores que los consumidores identifican al comprar en cualquiera de estas dos tiendas virtuales.

La diferenciación es importante en todo ámbito comercial, pero la percepción que tiene un usuario es bien cambiante, está dado por varios fenómenos como demográficos, socioeconómicos o también puede estar marcado por el marketing agresivo que las tiendas virtuales suelen utilizar en su etapa de introducción al mercado. Pero lo que todo comercio busca es la fidelidad del cliente y eso se genera a través de experiencias de compra y eso justamente es lo que se quiere analizar a partir de una revisión bibliográfica y un instrumento de análisis de mercado.

\section{DESARROLLO}

\section{El emprendimiento en la web}

Cada día son más personas que hacen del internet una herramienta imprescindible para su vida cotidiana, así también a nivel mundial las empresas por más pequeñas que éstas sean, utilizan y aprovechan la tecnología WEB. Analicemos brevemente 2 factores fundamentales en cuanto al emprendimiento en línea: la primera la idea de negocio y la segunda, la oportunidad de negocio. La primera está impulsada por el idealismo y la creatividad, y guiadas por la perseverante y su coste es bajo, mientras que la segunda debe existir un mercado que responda al producto o servicio que se propone como idea, además su coste es alto porque mueve un sinnúmero de recurso económicos, técnicos entre otros. Otros factores a considerar, según el nivel de implementación son: 


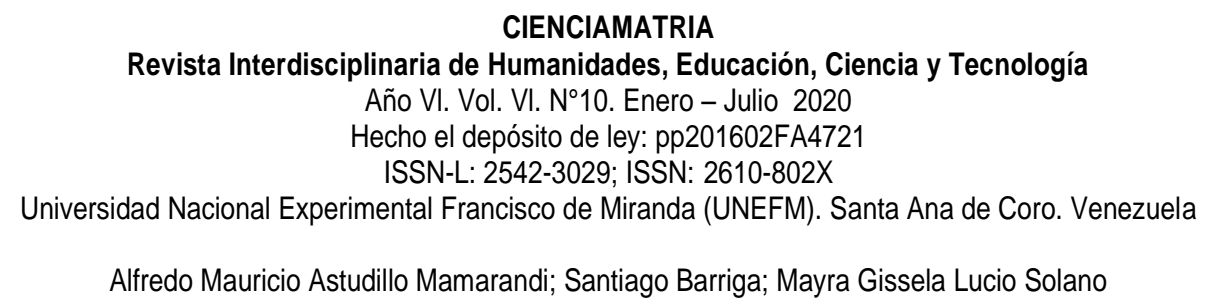

- La experiencia: está dado por el conocimiento íntegro del producto o servicio y su mercado objetivo

- Observación del entorno: se determina necesidades insatisfechas por la competencia o la misma empresa.

- Copiar lo que está hecho en otro entorno: repite experiencias ajenas pero adaptadas al entorno que se lo quiere enfocar.

- Franquicia: copia de un modelo exitoso

- Nuevas formas de vender: salir de un tradicionalismo de comercio y buscar nuevas formas de llegar al consumidor final

- Productos nuevos, tiene que ver con la innovación, algo que no existe en los mercados actuales.

Las fuentes de información son infinitas para generar ideas de negocio. Pueden estar en relación a la realidad del entorno, desarrollo económico-social de un país, e incluso, "basada en la intuición, creatividad, y experiencia profesional" (Gosende, 2014). Otros autores hacen mención de como conectar las ideas de negocio, a partir del conocimiento del entorno, las tendencias comerciales y tener información constante de la demanda y oferta de productos y servicios por internet. Los patrones que se toman en cuenta son:

- Montar un negocio donde hay demanda en internet. Como manera de ejemplo, con la ayuda de ciertas plataformas como Google Adwords, Trends se puede hacer un barrido de la demanda de ciertos productos en cualquier lugar del mundo y así se puede tomar decisiones de implementar un negocio.

- Buscar la debilidad de la competencia relevante. Mejorar el producto o servicio, aquí es necesario analizar el diseño de la página, la usabilidad, funcionalidades, la convertibilidad, hasta el posicionamiento en buscadores, esto destaca los puntos débiles y fuertes de la competencia. 


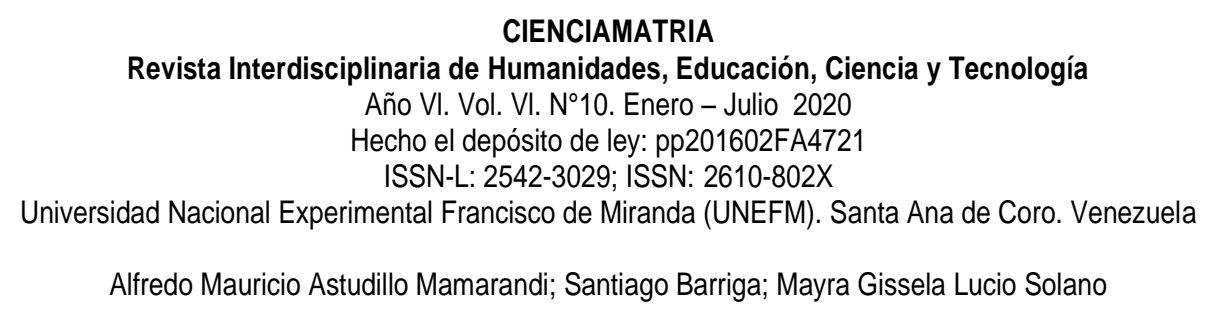

- Buscar pistas en internet, en dónde pueden estar los negocios. Por medio de la identificación de necesidades y experiencias de otros emprendedores se puede tener una información constante que ayude a crear la idea del negocio.

En cuanto a los 2 manifiestos para crear una idea de negocios, coinciden en algo muy importante como la experiencia y el conocimiento del entorno. Identifican de mejor manera las necesidades insatisfechas o nuevas necesidades y es allí donde debe nacer la innovación de lo identificado con una propuesta de valor que supere a la competencia o sea un factor diferenciador en el mercado.

\section{Características tecnológicas en el comercio electrónico}

El comercio electrónico desde un enfoque comercial se limita a una transacción entre el comprador y vendedor por medios digitales, pero atrás de esto, está toda una gama de funcionalidades articuladas en una infraestructura digital y administrativa que ayuda a efectuar de mejor manera dicha transacción. Es así que existen características únicas dentro de la tecnología que define al comercio electrónico como el camino al éxito o por lo menos al desarrollo prolongado de un negocio. Es necesario realizar un análisis de cada una de las dimensiones propuestas para que de aquello puedan surgir muchas posibilidades de comercio y sobre todo que dentro de este ámbito se puede construir intereses específicos de negocio: saber lo que piensan los consumidores, tener información primaria y efectiva en lo que se refiere a tráfico, segmentar según la necesidad (Laudon \& Traver , 2010). Y todo esto en el menor tiempo posible.

- Ubicuidad: libera al mercado del espacio físico, su disponibilidad no está limitado por tiempo ni espacio porque se crea un espacio de mercado y su medio común de uso son los dispositivos móviles.

- Alcance Global: trasciende los límites de la cultura y de naciones, su efectividad en reducción de costes puede ser significativa y conecta un sinfín de negocios en todo el mundo 


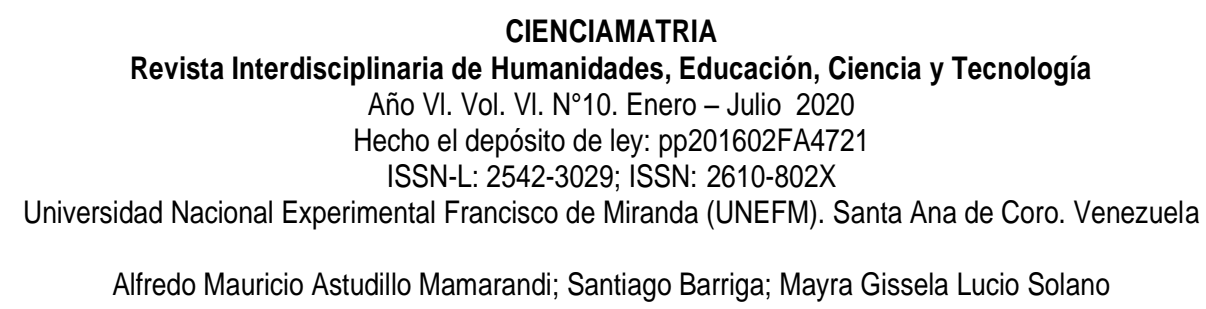

- Estándares universales: se refiere a lo técnico, tanto para el internet como para el comercio electrónico, son universales, por ejemplo, el lenguaje de programación, las bases de datos, el interfaz en cuanto a la usabilidad y un sin número de estándares que no cambian en naciones separadas geográficamente. Esto puede ayudar a reducir costes de búsqueda y de mercado

- Riqueza: esto no está dado por lo monetario, al contrario, tiene que ver con la complejidad de los mensajes comerciales, porque la WEB da la oportunidad de no atar los productos y/o servicios a una percha, sino que lo deja ver en diferentes perspectivas, apoyándose en la persuasión que tienen ciertos elementos como el video, la animación, el audio y el mensaje como tal.

- Interactividad: esto involucra necesariamente a los usuarios y sus experiencias y la tecnología logra eso con sus sofisticados medios de comunicación, el comerciante y el consumidor no se limitan a una transacción simple.

- Densidad de la información: la cantidad de información que puede llegar a tener un usuario puede ser abrumadora hasta caótica, pero si el usuario sabe bien lo que busca, esto puede funcionar a su favor, porque tiene a su alcance una variabilidad de ofertas de un mismo producto.

- Personalización / adecuación: se basa en las características individuales de cada comprador o grupos, la tecnología permite adaptar los mensajes, productos y servicios según los gustos y preferencias y compras anteriores.

- Tecnología social: la generación de contenidos no solo está ligada a los comerciales, sino también a los consumidores y no consumidores de un bien o servicio en línea y esto es posible gracias a las redes sociales.

Las características antes mencionadas pueden convertirse en barreras tanto de entrada como de salida para cualquier comercio electrónico, su complejidad está marcada por factores socioculturales, tecnológicos y hasta incumplimientos con ciertos parámetros 


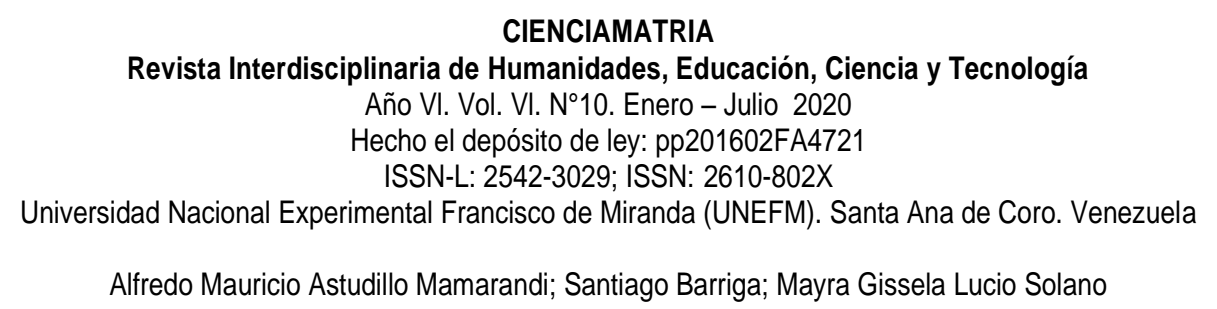

técnicos, o en mucho de los casos la ineficiencia del servicio, el cual es muy común en países en desarrollo.

\section{Tipos de comercio electrónico y modelos de negocio}

En el mundo del comercio electrónico, el proceso siempre será en línea y por medio de un interfaz, "el cliente visualiza y elige su producto, lo agrega al carrito de compras y pasa por caja para realizar el pago" (Rivas , 2014). Pero para llegar al fin de ese proceso es importante tener en cuenta la calidad de la información, seguridad, la navegabilidad del sitio web. Además, el entendimiento necesario para identificar qué tipo de comercio es, y el modelo en el que se basa el mismo, es importante este cuestionamiento, y que ayuda a entender el procedimiento de cada una de ellas, así como su comportamiento dentro del interfaz, ya que no es lo mismo navegar en una cuenta de bancaria online que en una empresa de servicios online. Cada una de ellas tiene su naturaleza, su contenido codificado para tipos de clientes específicos, entre otros.

\section{Tipos de comercio electrónico}

A continuación, se representa los tipos de negocios existentes en el mercado virtual, así tenemos:

- Entre empresas B2B, (Actúan como compradores, vendedores o proveedores)

- Entre empresa - consumidor B2C, (Vendes productos y servicios a clientes)

- Entre consumidor y consumidor C2C (realizan operaciones entre sí)

- Entre gobierno y consumidor G2C (interactuar entre ciudadano y gobierno)

- Entre empresas y gobierno B2G (optimiza procesos de negociación)

Todo tipo de comercio electrónico está relacionado con la naturaleza del mercado "quien vende a quien" (Laudon \& Traver , 2010). Pero estos autores además de mencionar algunos de los tipos de negocio antes descritos, también hace referencia a otros tipos de 


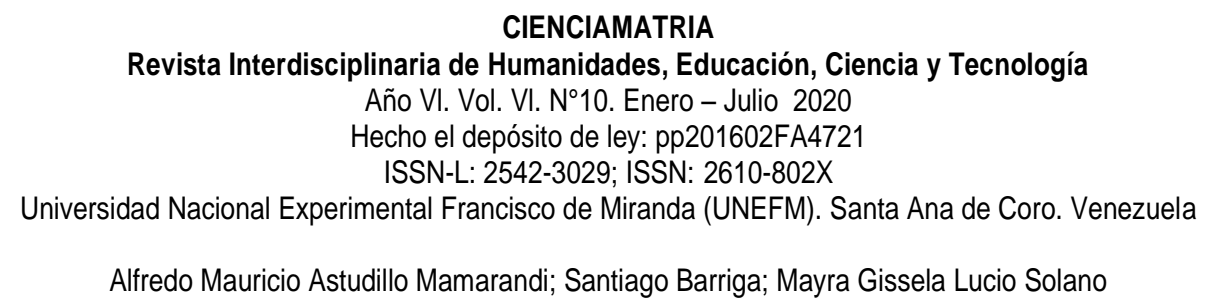

comercio electrónico como: P2P (comercio electrónico de igual a igual) en esencia este modelo permite compartir archivos de toda índole y recursos de computadora de forma directa y sin intermediarios. Por lo general utilizan "súper servidores" gran parte de este modelo se basa en movimientos ilegales y están en constante ataque de las autoridades. Otro es el Comercio Móvil (M-commerce), este se basa en la utilización única de dispositivos digitales inalámbricos para efectuar las actividades comerciales en la WEB y por ende las aplicaciones son las líneas de interfaz.

El B2E (negocio - empleado), es muy común su uso para mejorar el desempeño del empleado ya que atrae con ofertas especiales por pertenecer a la empresa (Lane, 2014). Sin duda existen diversos tipos de comercios electrónicos y cada uno se ajusta a la necesidad de los clientes, pero es fundamental que la información de los productos y servicios se presente de forma detallada, sus beneficios, el precio, entre otros. De eso depende el éxito de la misma desde un enfoque comercial.

\section{Modelo de negocios}

Dentro del mercado virtual existen muchos modelos que se aplica en el medio, pero a diferencia de los tipos de negocios el modelo está inclinado a generar ingresos y beneficios. A partir de la identificación del tipo de negocios, la empresa planifica el cómo llegar a sus clientes, la estrategia a implementar y el desarrollo de métodos de fidelización. También hay que tomar en cuenta que cada modelo de negocio tiene sus virtudes y puntos débiles, así como sus características propias, ninguna tiene la clave del éxito, al contrario, son rutas que marca el camino del emprendedor para desarrollar la idea de negocios. 


\section{Tabla 1.}

Modelo de negocios

\section{MODELO DESCRIPCIÓN CLAVES}

\begin{tabular}{|c|c|c|}
\hline $\begin{array}{l}\text { Ingresos por } \\
\text { publicidad }\end{array}$ & $\begin{array}{l}\text { Vende espacios publicitarios } \\
\text { a terceros, dentro de un } \\
\text { portal WEB, a través de } \\
\text { varios formatos como } \\
\text { banner, anuncios de pago o } \\
\text { patrocinio, y su alcance por } \\
\text { lo general es a largo plazo. }\end{array}$ & $\begin{array}{l}\text { - Cuotas muy altas de tráfico. } \\
\text { - Ingresos progresivos sin necesidad de que } \\
\text { aumente los costes. }\end{array}$ \\
\hline $\begin{array}{l}\text { Comercio } \\
\text { Electrónico }\end{array}$ & $\begin{array}{l}\text { Relación directa entre el } \\
\text { fabricante y el cliente y en } \\
\text { teoría reducción de precios; } \\
\text { tiene oportunidades de éxito } \\
\text { muy elevados si se } \\
\text { determina el nicho de } \\
\text { mercado adecuado. }\end{array}$ & $\begin{array}{l}\text { - Maximiza ganancias y reduce costos. } \\
\text { - Tiendas usables, persuasivas y amigables a } \\
\text { buscadores y redes sociales. } \\
\text { - Buena relación con proveedores. } \\
\text { - Promoción y marketing de la tienda online. } \\
\text { - Productos y/o servicios competitivos. } \\
\text { - Medios de pago. } \\
\text { - Atención al Cliente. } \\
\text { - Sistemas de logística eficiente. }\end{array}$ \\
\hline Afiliados & $\begin{array}{l}\text { Por lo general se basan en } \\
\text { tres modalidades, que son } \\
\text { por ingresos por visitas, } \\
\text { ingresos por re direccionar e } \\
\text { ingresos por venta, el pago } \\
\text { al afiliado va en proporción } \\
\text { al tipo de oferta. }\end{array}$ & $\begin{array}{l}\text { - Buscar nichos donde se pueda conseguir } \\
\text { altos niveles de visitas. } \\
\text { - Segmentar bien las campañas. } \\
\text { - Combinación de un buen contenido y } \\
\text { promociones que se muestre como ayuda } \\
\text { para el cliente. }\end{array}$ \\
\hline
\end{tabular}




\begin{tabular}{|c|c|c|}
\hline & & $\begin{array}{l}\text { - Ser transparente en los programas de } \\
\text { afiliación. }\end{array}$ \\
\hline Suscriptores & $\begin{array}{l}\text { Pagos periódicos por uso de } \\
\text { servicio y para su apertura } \\
\text { se necesita una fuerte } \\
\text { inversión. }\end{array}$ & $\begin{array}{l}\text { - Cuotas a definir con el usuario. } \\
\text { - Gran valor añadido. } \\
\text { - A mayor número de suscriptores menor o } \\
\text { igual coste total de la empresa. }\end{array}$ \\
\hline Suscriptores & $\begin{array}{l}\text { Al contrario de la anterior es } \\
\text { gratis, y sirve casi siempre } \\
\text { como enganche para ser un } \\
\text { suscriptor. }\end{array}$ & $\begin{array}{l}\text { - Su éxito está basado en los contenidos. } \\
\text { - Se debe notar la diferencia entre el premium } \\
\text { y freemuim. } \\
\text { - Mayor número de usuarios, para influir en su } \\
\text { decisión de compra. }\end{array}$ \\
\hline $\begin{array}{l}\text { Marca } \\
\text { personal }\end{array}$ & $\begin{array}{l}\text { Con la ayuda de las redes } \\
\text { sociales, los blogs, este se } \\
\text { ha hecho un modelo muy } \\
\text { común y es muy utilizado } \\
\text { por los freelance, porque } \\
\text { crea canales directos de } \\
\text { comunicación. }\end{array}$ & $\begin{array}{l}\text { - El internet es un intermediario. } \\
\text { - Se debe demuestra conocimiento, } \\
\text { experiencia, influencia y hasta imagen } \\
\text { personal. }\end{array}$ \\
\hline
\end{tabular}

Nota: el libro blanco del emprendimiento web, p. 60-75

Con la vertiginosa acogida que tienen las aplicaciones WEB, también da paso a otro modelo basado en la prestación de servicio así tenemos: el Software as a Service (Saas): El "Software como Servicio" está relacionado con todo el software de aplicaciones de gestión ubicadas en la "nube". Este permite que personas y pymes accedan a herramientas de gestión en áreas como contables, de facturación, CMR, salud, estado físico, etc. 
Y, por último, el Proveedor de soluciones y Contenidos (Api): está basado en redes de distribución compartida por proveedores de herramientas y aplicaciones, las mismas pueden ser utilizadas para otros servicios o desarrollos de otras aplicaciones (Santos , 2015).

\section{El tráfico}

En la red, el tráfico es el factor predominante para cerrar una venta o generar negocio en línea mientras más personas visiten un comercio electrónico mayor será la ventaja de la conversión, Quique Mora (2016) lo llama, la viabilidad económica desde una visión cualitativa, porque reflexiona sobre 2 claves de análisis dentro de este tema, a la nueva demanda y a la especialización de oferta de bienes y servicios. El mismo autor hace énfasis en dos factores diferenciadores para el éxito de dicha reflexión: Ofrecer algo distinto a lo que existe en el mercado y conseguir visitas que puedan convertirse en clientes. Además, algo muy importante que destaca, es determinar al cliente ideal, reconocer sus problemas actuales, como la falta de tiempo, distancia de compra, facilidad de pago, etc., y en base a lo mencionado hacer una propuesta de valor única enfocado en sus necesidades. Si se identifica a ese cliente y se determina el porcentaje de ganancia en cada uno pasa a la segunda clave, la cual está fundamentada en el tráfico para alcanzar la viabilidad, eso se traduce en cuántas visitas se debe tener para concretar una venta.

Por otro lado, en numerosos estudios sobre el comercio electrónico en economías en desarrollo, Boateng, Molla y Heeks, coinciden en aspectos como: difusión de la innovación (DOI), el comportamiento planificado (TPB) y la aceptación de la tecnología (TAM) que siempre son utilizadas en combinación, dependiendo del entorno, tecnología, empresa, entre otros (Molla, 2004) factores que identifican con claridad al usuario ideal para generar tráfico WEB en colaboración con otras estrategias muy utilizadas en el medio como son: 


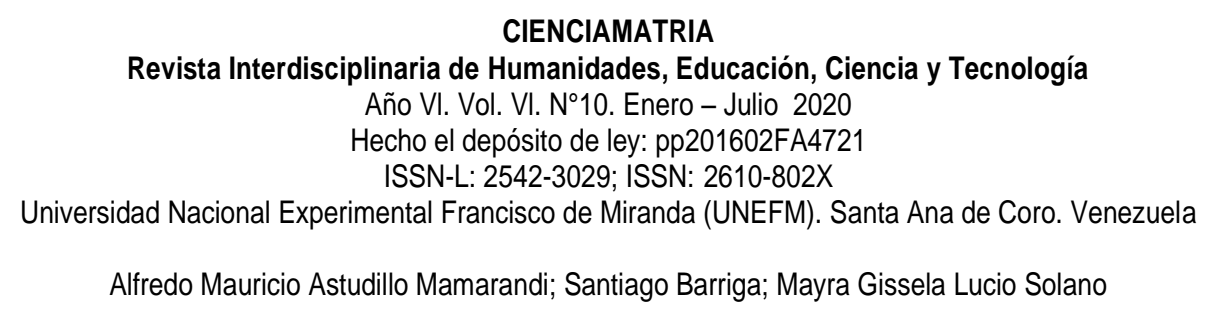

- Tráfico de Marca, por medio del URL del negocio, muy común cuando se realiza campañas de marketing directo.

- Tráfico de Referencia, utiliza enlaces y casi siempre son utilizados en las redes sociales, también están los blogs o web en los que se menciona al negocio. Muchas de las veces en el marketing de guerrilla, la prensa. Todo lo que pueda dar un enlace e información del negocio y sea convertido en visita.

- Tráfico de búsqueda, se apoya de las plataformas de búsqueda que sean gratuitas o de pago, con la particularidad de esgrimir palabras claves significativas y relevantes.

Este último utiliza estrategias de marketing diferenciadas como es el SEO y SEM. EI primero conlleva técnicas más orgánicas y tiene 2 parámetros de actuación el SEO ONPAGE (elementos internos como keywords, URLs, tiempo de carga) y el SEO OFF-PAGE (elementos externos como enlaces. CRT. Presencia en redes). El segundo es de pago (posicionamiento en buscadores), campañas mediante anuncios en el Google AdWords y/ Google Shopping (Maciá, 2015).

Desde el punto de vista de las diferenciaciones entre SEM y SEO, están: las palabras claves (SEM limitado - SEO ilimitado), en costos SEM tiene costos fijos, conlleva mayor tiempo y son poco flexibles y SEO tiene costos variables, con menor tiempo y muy flexibles en posicionamiento de búsqueda. En cuanto a: contenidos el SEO valora la calidad y extensión de texto, mientras que el SEM tiene un texto más comercial. En la vialidad SEO, no se garantiza estar entre los primeros puestos en los buscadores, mientras que el SEM ocurre lo contrario desde el primer momento. Y en Medición el SEO es muy complejo para medir por no tener datos actuales, mientras que en SEM mide en vivo, gracias a los datos de coste e ingresos (IDENTO, 2017). 


\section{Seguridad}

En el comercio electrónico, la seguridad es el principal aspecto a tener en cuenta, tanto en los proveedores como en los consumidores, porque en ello se transforma la confianza de una transacción (Parrilla, 2016). Lo primordial al momento de efectuar una transacción en la web, es el medio seguro que ofrece la página para el pago. Esto se logra con implementaciones de certificados SSL (Secure Socket Layer) un protocolo de seguridad que transporta la información de forma cifrada o encriptado y para identificarlo solo vasta que aparezca un ícono de candado de color verde, la barra de exploración y al inicio de la dirección URL las siglas del protocolo HTTPS (Certsuperios, 2015).

Según expertos el "Phishing" y el "Spam" son 2 de las amenazas fraudulentas más recurridas en el comercio electrónico, porque se sirven de un sinfín de tácticas para conseguir información confidencial como contraseñas, datos de cuentas, códigos Pin, etc. Por eso es muy importante tener habilitado software antivirus, antimalware y actualizar el sistema operativo. Otro factor a tomar en cuenta en seguridad es, aplicar el test de penetración y ofrecer pasarelas de pago como PAYPAL. Este sistema permite a los usuarios concluir la transacción a través del internet sin compartir la información financiera con la empresa o el destinatario del pago.

\section{Sistemas de pago}

A nivel mundial existe sistemas de pago muy marcados como son: el efectivo, las tarjetas de crédito, tarjeras de débito, la criptomonedas (no es legal en todo el mundo), las transferencias bancarias y el dinero electrónico, cada una con su característica propia. Pero en el ámbito ecuatoriano con respecto al comercio electrónico, se generan grandes conflictos, los factores pueden variar: la desconfianza al uso de estos medios, el desconocimiento tecnológico, la amplitud de tiendas locales al uso de estos medios de pago no tradicionales, entre otros. Esto causa problemas "especialmente en la interacción de pagos a través de distintas tarjetas de crédito" (Korntheuer, 2017). 
La integración del botón de pago en cualquier comercio electrónico, no está dada por las plataformas que sirven para desarrollar dicha actividad, caso explicito, ni Magento, Shopufy, Prestashop, Wordpress y otros gestores de contenido no ofrecen esa integración con el sistema bancario del Ecuador. Sin embargo, existen los Gateway de pago, empresas intermediarias de negociación con operadoras de crédito y bancos, es muy útil por su seguridad, ya que los datos del usuario se almacenan en un único sistema y este puede ser reutilizado en futuras compras. No tiene que volver a cargar los datos cada vez que lo utilice o analizar si confía o no en el sitio web para dar los datos de la tarjeta, tal situación a juicio de Urueta Vélez (2019), la tecnología permite contribuir en el desarrollo de las empresas, propiciando mayor efectividad en sus procesos. A continuación, se presenta algunas opciones para los pagos on-line:

\section{Tabla 2.}

Botones o sistemas de pago que hay en el Ecuador

TIPO

Paypal

PayPhone

Kushki

\section{CARACTERÍSTICAS}

Es el más utilizado Posee la mayor confianza en el mercado.

Comisiones muy altas para el mercado local.

Es un sistema internacional sin representación en el país.

Se necesita tener una cuenta en el exterior para habilitar el botón de pago.

Funciona en la web y en sistemas APP y es gratuito.

Genera cobros seguros con interés o sin él.

Precios de comisión de servicio son bajos.

No tiene representación en el país.

Tiene vigencia en el país, trabaja con Visa y MasterCard.

Divide la compra en cuotas (diferido).

La comisión es variada y en constante cambio monetario. 


\begin{tabular}{ll}
\hline & Recarga las comisiones automáticamente en las cuotas. \\
& Tiene modelos de integración con: Woo-commerce. Magento, \\
Prestashop, Vetex y & Shophifi. \\
& Su costo transaccional es de aproximadamente el 1.5\%+0.5 ctvos \\
+ IVA. & Trabaja bajo códigos de validación con respecto al cliente. \\
PayClub & Es un canal adicional de venta. \\
& Funciona solo con APP. \\
Tiene experiencia en mercados sudamericanos. \\
Es multiplataforma (Web, Mobile y APP), ofrece antifraude. \\
Tiene certificados PCI DSS 3.2. \\
Funciona bajo registro, Trabaja con el Banco del Pichincha. \\
Tiene una gran gama de medios de pago y aleados financieros. \\
Método fácil y seguro. \\
Es el menos utilizado entre los usuarios. \\
Muchos procesos por cumplir. \\
Proporciona mayor inseguridad entre los clientes y el negocio. \\
Cantra entrega
\end{tabular}

Nota: Tomado de monkeyplus ${ }^{1}$

La base teórica presentada, hace mención a muchos factores que coadyuvan al mejoramiento de un comercio justo, rápido y más equitativo, así como también presenta riesgos en el momento de generar pagos de productos y servicios en línea, por lo que es importante hacer uso de herramientas de investigación de mercados y aplicarlas en 


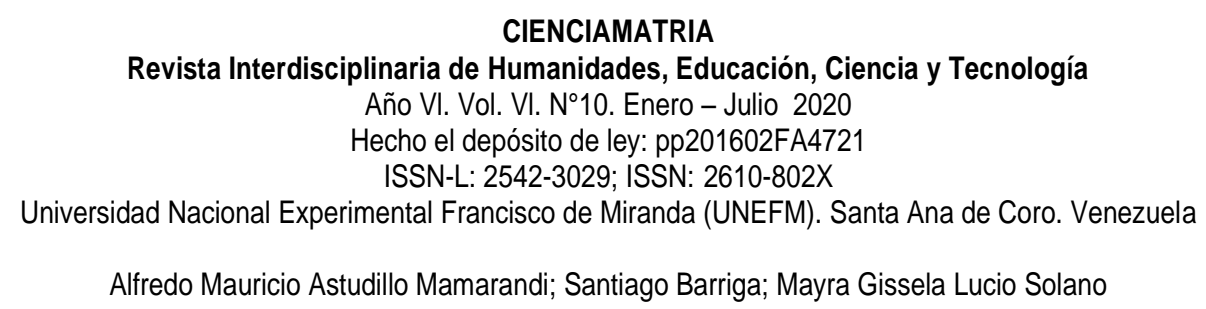

consumidores WEB cotidianos. Por lo tanto, este documento pretende poner en marcha un instrumento de recolección de datos cualitativos, para analizar el tiempo de aprendizaje, percepción y aceptación de las tiendas en línea, a diferencia de estudios de la misma naturaleza es que se lo realizara de producto poco tradicionales en la compra y venta virtual.

\section{METODOLOGÍA}

Para entender cómo opera un mercado virtual y como se mantiene la percepción de un servicio en línea se toma en cuenta una metodología no experimental observacional descriptivo transversal, a partir de datos cuantitativos (Sampieri, 2010), el Focus Groups (o grupo de discusión) muy usada en la investigación de mercados el cual sirve para recolectar información primaria. Mediante este modelo un moderador intenta develar la motivación de un comprador (Kotler \& Keller, 2012).

Para la ejecución del esquema metodológico e instrumental fue necesario tomar ciertas características para el escogimiento de los panelistas, la más importante, que tenga experiencia dentro del comercio electrónico y que tengan un poder adquisitivo medio alto, además de tener un ingreso fijo. En el estudio participaron personas que laboran en instituciones pública y privadas de la ciudad de Riobamba y se escogido dos tiendas virtuales ecuatorianas que promocionar y vender productos de primera necesidad (http://ianser.ec/ - https://supereasy.ec). El rango de edad de los panelistas oscilaba entre los 31 a 42 años de edad, en cuanto al tamaño de la muestra, intervinieron 18 personas de ambos sexos. Tiempo estimado del desarrollo del instrumento aplicado en la investigación fue de 45 minutos y fecha de ejecución, el mes de enero del 2019.

La prueba se plantea en dos escenarios: El primer escenario, es un estudio comparativo de usabilidad, interfaz y seguridad dentro del entorno virtual, en donde se le pide a cada uno de los panelistas observar detenidamente las pantallas y vínculos de las tiendas virtuales, se les da un lapso de 1 minuto por cada tienda, para fines de evaluación se 
asigna un número a cada página (http://ianser.ec/, con el número 1 y https://supereasy.ec, con el número 2) y se les solicito registrar sus respuestas en una ficha adicional (encuesta semiestructurada) con respecto a las preguntas efectuadas.

En el segundo escenario, por la objetividad de la prueba aplicada, se les solicita a los participantes completar de forma individual, su percepción a las características que considere relevante en una compra en línea; entre ellas se planteó las siguientes variables: Seguridad, Rapidez, Diversificación, Precios, Marca y/o Publicidad.

\section{RESULTADOS Y DISCUSIÓN}

Luego de la revisión y análisis de los resultados del Focus Group se presenta de forma sintetizada los criterios tomados en cuenta por los panelistas.

\section{Tabla 3.}

Tamaño de la muestra

\begin{tabular}{llll}
\hline Variable & $N^{\circ}$ de participantes & Porcentaje & $\begin{array}{l}\text { Porcentaje } \\
\text { acumulado }\end{array}$ \\
\hline Mujeres & 12 & 66,7 & 66,7 \\
Hombres & 6 & 33,3 & 100,0 \\
TOTAL & 18 & 100,0 & \\
\hline
\end{tabular}

Como se mencionó en la metodología, se reunió a un grupo homogéneo en ingresos y actividad laboral, en cuanto a lo heterogeneidad se muestra que la mayoría de panelistas fueron mujeres con un $67 \%$ aproximado, los factores pueden ser varios pero se deduce según lo observado, que las mujeres son más versátiles en compras por internet, mientras que los hombres lo hacen por preferencia, es así que cuando se les preguntó que si habían hecho alguna compra en línea en el último mes, las mujeres mencionaron 


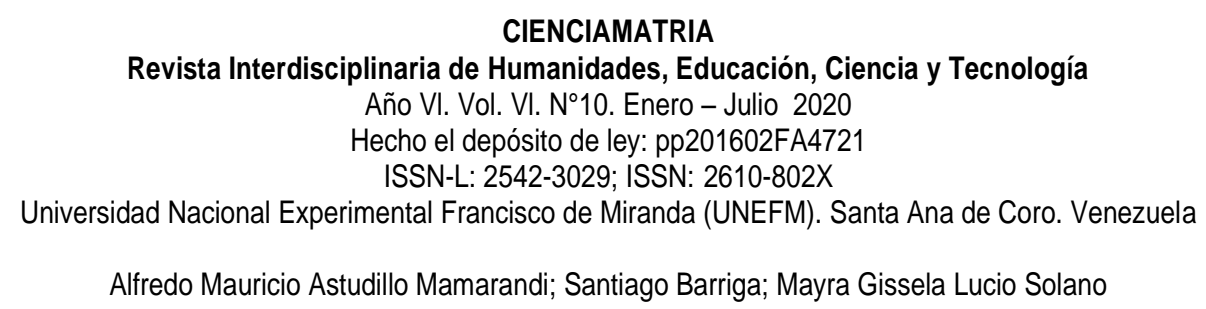

que sí y en una frecuencia mayor a dos veces, mientras que los hombres en su mayoría no lo habían hecho.

Los panelistas desde sus entendimientos, identifican como un tipo de negocio B2C, y se llegó a esa conclusión por los siguientes factores: los panelistas aseguran en un $94.00 \%$ que está teniendo relación directa con la tienda, sin tener que estar en forma física en ella, además la tienda presenta un diseño muy marcado en cuanto a nichos de mercado, ya que se identifica con claridad el objetivo de la misma y a quien está dirigido los productos que oferta, además, desde una perspectiva general coinciden en más de un $80 \%$ que las tiendas son usables, persuasivas y amigables.

Dentro del estudio comparativo que corresponde al primer escenario, de forma general la percepción que los panelistas tienen con respecto a las tiendas virtuales mostradas, se menciona lo siguiente:

Luego de observar y manipular la interfaz de las 2 páginas WEB ¿Qué tienda virtual tiene mejor organización con respecto a los productos ofertados? Según los panelistas ianser.ec supera con un $56 \%$, la mayoría coincide en que las fotos de los productos hicieron fácil el reconocimiento y su organización por utilidad o uso, como factor diferenciador, mientras que a la tienda virtual supereasy.ec obtuvo un $44 \%$ ya que el usuario demoró en reconocer la organización porque el primer pantallazo muestra botella de agua, pero luego identificaron la organización de los productos en el menú de categorías. ¿En qué tienda se puede identificar rápidamente sus categorías por productos? Los panelistas mencionan que la ayuda de iconos e incluso el color utilizado, fue un factor determinante para identificar con rapidez las categorías. La tienda virtual ianser.ec con un $83 \%$ de aceptación, aplica la intuición por color y eso para los panelistas es fundadamente porque se genera una organización en base a cromática y esto apoyado con imágenes que vinculen lo antes mencionado.

En cuanto a navegabilidad se preguntó ¿cuál de las tiendas tiene una buena disposición de botones? Con casi $90 \%$ de aceptación, iancer.ec tiene una buena navegabilidad, 


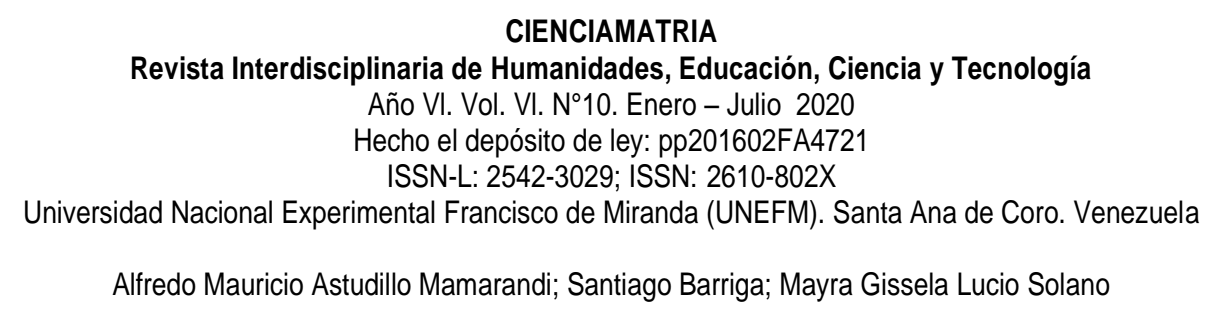

según la percepción de los panelistas, insisten que los factores determinantes para una buena navegabilidad son la disposición de botones llamativos, que tengan iconos y textos, además de la ayuda de menús desplegables hace más fácil el recorrido por la tienda virtual. Además, al navegar por las categorías, los productos, carro de compras, los panelistas no encontraron dificultades para regresar a la página principal o dirigirse a otros productos, esto da muestra que las dos tiendas virtuales tienen diseños web persuasivos y amigables con el usuario, haciendo que la interfaz sea más fácil y su experiencia de compra sea muy agradable.

Para entender de mejor manera la usabilidad, navegabilidad y lo persuasivo que llegaron hacer cada tienda virtual, se le dio a cada panelista un listado de 10 productos, los cuales debían ser llevados al carro de compras de cada tienda y se lo debía hacer por separado, llegando a determinar que más del $70 \%$ de los panelistas lo logro en menos de 2 minuto hacerlo en iancer.ec. mientras que en supereasy.ec, se superó los 2 minutos. En términos generales manifiestan que se debe a la organización de los productos y que en muchos de los casos se les dificultaba entender en donde se encontraban como usuarios. Algo muy importante a tomar en cuanta al desarrollar un proyecto de estas características, ya que no solo es lo visible lo que llama la atención sino también lo funcional que puede ser. En lo que tiene que ver con sistemas de pago, en primera instancia en la pantalla inicial de cada tienda virtual solo ianser.ec mostraba los sistemas de pago y no limitaba al uso de tarjetas, al contrario, diversificaba las líneas de pago así promovía en el usuario una mayor confianza a la hora de elegir esta tienda virtual. Algo interesante que hay que rescatar es que los panelistas hicieron uso del carro de compras y en las dos tiendas existía un estricto registro para realizar el pago de los productos seleccionados, y la mayoría de personas coincidían que es bueno porque está dando muestras de la seriedad de la plataforma.

Aquí cabe un análisis desde el ámbito técnico, el uso del botón de pago y la diversidad del mismo en los comercios electrónicos ecuatorianos, es muy complicada, para su 


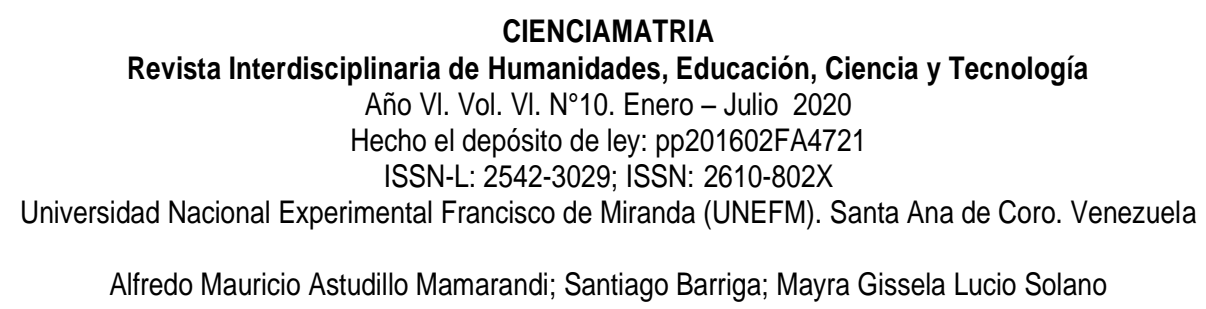

aplicación. En el marco contextual de este documento se hizo una revisión de los Gateway de Pago más aceptados o reconocidos en el ámbito del comercio electrónico en nuestro país y se identificó que no existen formas de integrar pagos a través de distintas tarjetas de crédito y débito en un solo botón. Definitivamente, eso causa problemas, porque normalmente se necesita de plataformas distintas; una conexión para Visa/MasterCard y otra conexión para Diners (Payclub). En cuanto a la diversidad de pagos en línea en la ciudad de Riobamba hay una preferencia marcada con respecto al uso de tarjetas de crédito por su rapidez, seguridad y que los rubros más importantes a pagar son alimentos y servicios básicos (Astudillo \& Ochoa, 2017).

Los panelistas mencionaron algo muy importante y que para ellos es novedoso dentro del mercado de productos de primera necesidad y es que ninguna de las dos tiendas virtuales tenia productos en oferta o descuento, peor aún en presentación tipo canasta de productos y que eso no es bueno desde su percepción ya que esta es una forma de enganchar la compra. Según ellos, el tener acceso a canastas elaboradas, evita la búsqueda innecesaria y se centra en lo que se necesita.

En el segundo escenario, en el estudio de variables la percepción a las características que se considera relevante en una compra en línea, supieron mencionar lo siguiente:

Lo más relevante que los panelistas ponen a consideración antes de realizar una compra, es la publicidad con un $94 \%$ aproximadamente, seguida de la diversificación y precio quienes comparten un $75 \%$ de preferencia, esto marca una pauta muy importante, en el momento de lanzar la tienda virtual, la publicidad que se debe generar en torno a la tienda virtual debe ser una prioridad, los canales de comunicación y las campañas deben proponer una evangelización del servicio.

\section{CONCLUSIÓN}

Al implementar un instrumento como el Focus Group para determinar la percepción del usuario riobambeño en la interacción web, ayudo a entender varios factores como: que 


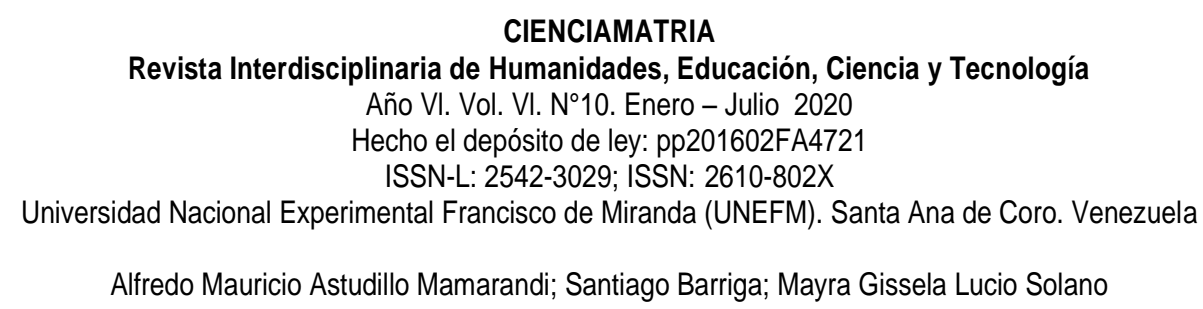

las mujeres son más propensas al uso de compra en línea, además una variable a considerar es la publicidad y el contenido de estos negocios en línea, para atraer a potenciales clientes, por otro lado, la diversificación, el constante cambio de presentaciones, combinaciones de productos y versatilidad de los mismo, las políticas claras en cuanto a sistemas de pago y seguridad de la información, entre otras.

Los panelistas rescatan, factores diferenciadores de cada tienda, como la presentación de los productos, la diversificación de los mismos y los costos, también se mencionó que para los panelistas es nuevo este tipo de negocio, el factor más primordial era el desconocimiento, pero si se lo implementara se arriesgarían el utilizarlo; y algo más a destacar es que, no solo la presentación de los productos conlleva a una experiencia, sino todo el proceso desde que se escoge el producto hasta que llega al consumidor final, pero sin embargo para la mayoría le parecía novedoso este sistema de compra de productos de primera necesidad.

Algo muy importante a tomar en cuenta como modelo de negocios, es que la información que se presente en cuanto al sistema de pagos es primordial, la diversidad dentro de este tema debe ser tomado en cuenta y sustentado, porque de esto dependerá un retorno del cliente, al sentirse respaldado por lo que necesita y por la seguridad que le brinda en el momento del pago. En cuanto a la presentación de los productos al no presentar como canastas no ocasiona ninguna dificultad, lo que se tomó en cuenta, es el tiempo que tiene el usuario para revisar todos los links que tiene la tienda virtual.

Por último Las mujeres toman una iniciativa mayor con respecto a comprar en línea, los factores más relevantes para la compra a nivel general, está la publicidad, seguida de la diversificación y el precio, además la organización dentro de la tienda virtual se torna vital para el desempeño de la misma y cierre de una compra, además están relacionadas directamente del cómo se presenten las categorías de productos ofertados y su dificultad de entradas y salidas de cada categoría está marcado por la menor dificultad posible, es decir que es muy importante que el usuario se sienta como si estuviera en una tienda 


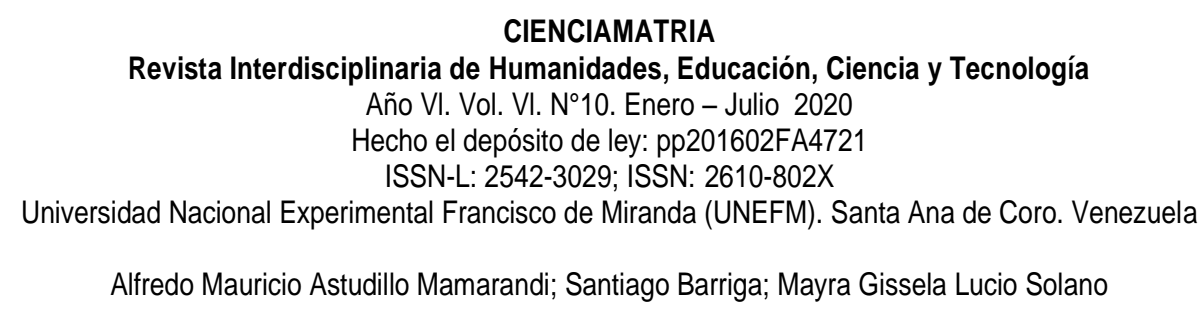

física, y eso recae en el estudio de la usabilidad y accesibilidad de cada tienda, esto influye en la posibilidad de que el comercio electrónico explore alternativas de compras para los consumidores, tal como lo destacan Anderson, Graterol \& Zabeta (2018), cuando destacan las bondades que proporciona el comercio electrónico, el cual debe gestarse en un marco donde se promueva la seguridad como indicador de confianza al cliente.

\section{REFERENCIAS CONSULTADAS}

1. Anderson, C., Graterol, E., \& Zabeta, M. (2018). LA TRIBUTACION DEL COMERCIO ELECTRÓNICO EN EL MUNICIPIO MIRANDA DEL ESTADO FALCÓN. IUSTITIA SOCIALIS, 2(2), 77-87. Recuperado de http://fundacionkoinonia.com.ve/ojs/index.php/lustitia Socialis/article/view/90/75

2. Astudillo, A., \& Ochoa, P. (17 de 08 de 2017). Repositorio Digital UNACH. Obtenido de http://dspace.unach.edu.ec/handle/51000/4205

3. Certsuperios. (14 de 07 de 2015). Certificados de Seguridad para el E-commerce. Obtenido de https://www.certsuperior.com

4. Gosende, J. (2014). El Libro Blanco del Emprendedor WEB. Madrid: ANAYA.

5. IDENTO. (20 de 03 de 2017). MARKETING ONLINE, SEO Y DISEÑO WEB. Obtenido de Diferencias entre SEO y SEM: https://www.idento.es/blog

6. Korntheuer, R. (24 de 05 de 2017). Ecuador: Comercio electrónico y formas de pago. Obtenido de seo-quito.com: https://seo-quito.com

7. Kotler, P., \& Keller, K. (2012). Dirección de Marketing. México: PEARSON.

8. Laudon, K., \& Traver, C. (2010). E-commerce. México: PEARSON.

9. Lane, A. (2014). Los 5 tipos de comercio electrónico. Obtenido de shopify.com: https://es.shopify.com/blog

10. Maciá, F. (2015). Marketing Online 2.0. Madrid: ANAYA. 


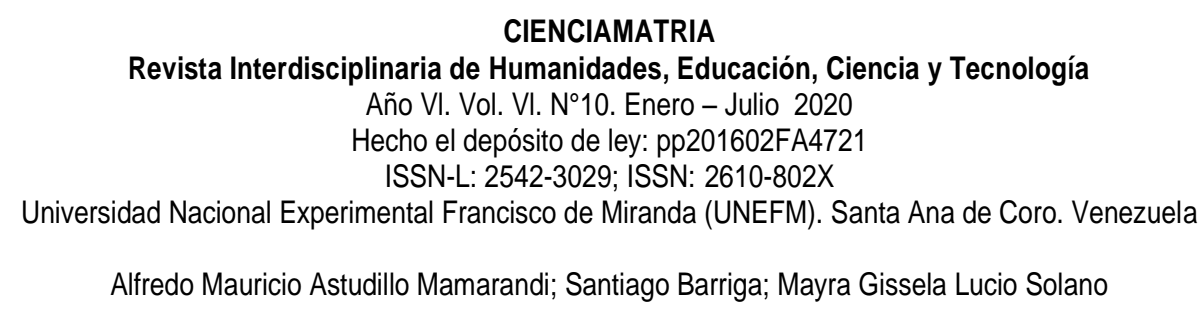

11. Mora, Q. (13 de 02 de 2016). infoautonomos.eleconomista.es. Obtenido de Viabilidad económica de tu idea de negocio online: http://infoautonomos.eleconomista.es

12. MONKEYPLUSBC, agencia ecuatoriana de marketing digital, especializada en servicios web, los cuales hacen un análisis de los botones de pago en el Ecuador. Blog disponible en https://monkeyplusbc.com/blog-marketing-digitalecommerce/boton-de-pago-para-una-tienda-online-ecommerce-en-ecuador.html Castañer, J. (28 de 02 de 2014). Junta de Planificación / análisis de costo beneficio. Estudios Técnicos INC. Obtenido de http://gis.jp.pr.gov

13. Parrilla, L. (03 de 03 de 2016). Universidad Carlos III de Madrid. Obtenido de Repositorio institucional e-Archivo: https://e-archivo.uc3m.es

14. Rivas, C. (14 de 11 de 2014). Comercio Electrónico - CITUNT. Obtenido de Krivas: http://krivas-ecommerce.blogspot.com

15. Sampieri, F. \&. (2010). METODOLOGÍA DE LA INVESTIGACION (QUINTA ed.). MEXICO: McGraw-Hill.

16. Santos, J. (08 de 06 de 2015). Infoautónomos. Obtenido de Modelos de Negocio en Internet: http://infoautonomos.eleconomista.es

\section{CONSULTED REFERENCE}

1. Anderson, C., Graterol, E., \& Zabeta, M. (2018). THE TAXATION OF ELECTRONIC COMMERCE IN THE MIRANDA MUNICIPALITY OF THE FALCÓN STATE. IUSTITIA SOCIALIS, 2 (2), 77-87. Recovered from http://fundacionkoinonia.com.ve/ojs/index.php/lustitia Socialis/article/view/90/75

2. Astudillo, A., \& Ochoa, P. (17 of 08 of 2017). UNACH Digital Repository. Retrieved from http://dspace.unach.edu.ec/handle/51000/4205

3. Certsuperios. (14 of 07 of 2015). Security Certificates for E-commerce. Obtained from https://www.certsuperior.com

4. Gosende, J. (2014). The White Paper of the WEB Entrepreneur. Madrid: ANAYA. 


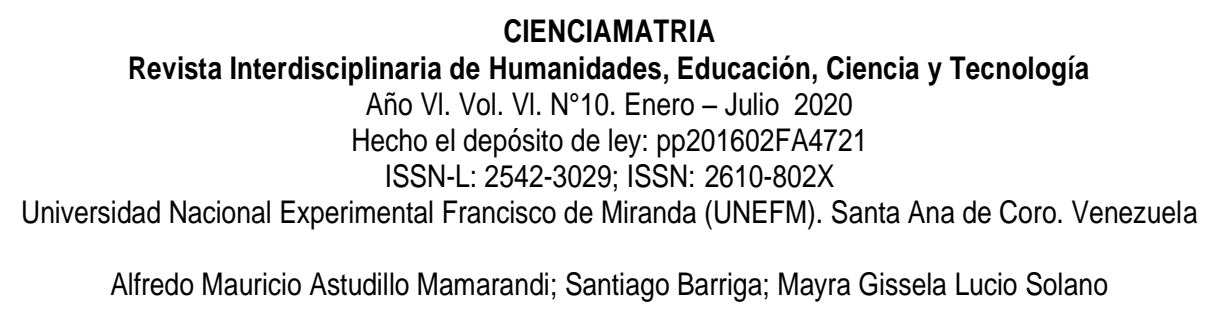

5. IDENT. (20 of 03 of 2017). ONLINE MARKETING, SEO AND WEB DESIGN. Obtained of Differences between SEO and SEM: https://www.idento.es/blog

6. Korntheuer, R. (05 of 05 of 2017). Ecuador: Electronic commerce and payment methods. Obtained from seo-quito.com: https: // se o-quito.com

7. Kotler, P., \& Keller, K. (2012). Marketing direction . Mexico: PEARSON.

8. Laudon, K., \& Traver, C. (2010). E-commerce Mexico: PEARSON.

9. Lane, A. (2014). The 5 types of electronic commerce. Obtained from shopify.com: https://es.shopify.com/blog

10. Maciá, F. (2015). Online Marketing 2.0. Madrid: ANAYA.

11. Mora, Q. (13 of 02 of 2016). infoautonomos.eleconomista.es. Obtained from the economic viability of your online business idea: http://infoautonomos.eleconomista.es

12. MONKEYPLUSBC, Ecuadorian digital marketing agency, specializing in web services, which analyzes the payment buttons in Ecuador. Blog available at https://monkeyplusbc.com/blog-marketing-digital-ecommerce/boton-de-pagopara-una-tienda-online-ecommerce-en-ecuador.html Castañer, J. (28 of 02 of 2014). Planning Board / cost benefit analysis. Technical Studies INC. Retrieved from http://gis.jp.pr.gov

13. Parrilla, L. (03 of 03 of 2016). University Carlos III of Madrid. Obtained from Institutional Repository e-Archive: https://e-archivo.uc3m.es

14. Rivas, C. (14 of 11 of 2014). Electronic Commerce - CITUNT. Obtained from krivas: http://krivas-ecommerce.blogspot.com

15. Sampieri, F. \&. (2010). RESEARCH METHODOLOGY (FIFTH ed.). MEXICO: McGrawHil.

16. Santos, J. (08 of 06 of 2015). Autonomous info. Obtained from Internet Business Models: http://infoautonomos.eleconomista.es

17. Urueta Vélez, L. (2019). Estrategias de enseñanza y el uso de las tecnologías de información y comunicación en las instituciones educativas departamentales en el 


\section{CIENCIAMATRIA}

Revista Interdisciplinaria de Humanidades, Educación, Ciencia y Tecnología

Año VI. Vol. VI. №10. Enero - Julio 2020

Hecho el depósito de ley: pp201602FA4721

ISSN-L: 2542-3029; ISSN: 2610-802X

Universidad Nacional Experimental Francisco de Miranda (UNEFM). Santa Ana de Coro. Venezuela

Alfredo Mauricio Astudillo Mamarandi; Santiago Barriga; Mayra Gissela Lucio Solano

Municipio Zona Bananera - Colombia. Revista Arbitrada Interdisciplinaria Koinonía, 4(7), 185-201. doi:http://dx.doi.org/10.35381/r.k.v4i7.200

18. Urueta Vélez, L. (2019). Teaching strategies and the use of information and communication technologies in departmental educational institutions in the Municipality of Bananera Zone - Colombia. Interdisciplinary Arbitrated Review Koinonía, 4 (7), 185-201. doi: http: //dx.doi.org/10.35381/r.k.v4i7.200 